\title{
Intestinal Obstruction due to Complete Transmural Migration of a Retained Surgical Sponge into the Intestine
}

\author{
Takashi Kato $^{a}$ Koji Yamaguchi $^{d} K_{\text {Koji Kinoshita }}{ }^{a}$

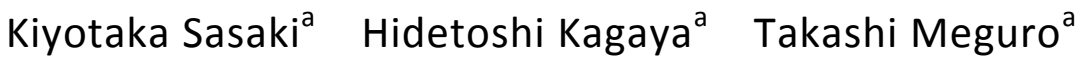 \\ Takayuki Morita $^{b}$ Toshiyuki Takahashic $^{c}$ Nagara Tamaki $^{\mathrm{e}}$ \\ Shoichi Horita ${ }^{a}$ \\ Departments of ${ }^{\mathrm{a}}$ Internal Medicine, ${ }^{\mathrm{b}}$ Surgery and ${ }^{\mathrm{c}}$ Pathology, Hokkaido \\ Gastroenterology Hospital, Sapporo, d Department of Surgery, Japanese Red \\ Cross Kitami Hospital, Kitami, and ${ }^{\mathrm{e}}$ Department of Nuclear Medicine, Hokkaido \\ University Graduate School of Medicine, Sapporo, Japan
}

\section{Key Words}

Retained surgical sponge - Gossypiboma $\cdot$ Textiloma $\cdot$ Intestinal obstruction $\cdot$ Ileus

\begin{abstract}
A 56-year-old woman with a history of gynecological surgery for cervical cancer 18 years previously was referred to our hospital for colicky abdominal pain, nausea and vomiting. Intestinal obstruction was diagnosed by contrast-enhanced computed tomography (CT) which showed dilation of the small intestine and suggested obstruction in the terminal ileum. In addition, CT showed a thick-walled cavitary lesion communicating with the proximal jejunum. ${ }^{18} \mathrm{~F}$-fluorodeoxyglucose positron emission tomography showed abnormal uptake at the same location as the cavitary lesion revealed by CT. The patient underwent laparotomy for the ileus and resection of the cavitary lesion. At laparotomy, we found a retained surgical sponge in the ileum $60 \mathrm{~cm}$ from the ileocecal valve. The cavitary tumor had two fistulae communicating with the proximal jejunum. The tumor was resected en bloc together with the transverse colon, part of the jejunum and the duodenum. Microscopic examination revealed fibrous encapsulation and foreign body giant cell reaction. Since a retained surgical sponge without radiopaque markers is extremely difficult to diagnose, retained surgical sponge should be considered in the differential diagnosis of intestinal obstruction in patients who have undergone previous abdominal surgery.
\end{abstract}




\section{Introduction}

Despite improvements in the development of surgical techniques, retained surgical sponges remains a serious problem. Nonspecific clinical presentations and diverse imaging features often create difficulty making an accurate preoperative diagnosis [1]. Pathologically, retained surgical sponges are associated with two types of foreign body reactions. One is an aseptic fibrous response that creates adhesions and encapsulation; this results in the formation of a foreign body granuloma, usually after a silent clinical course. The second response is exudative in nature and leads to abscess formation with or without secondary bacterial infection. In the latter scenario, the retained surgical sponge may present with abdominal pain, abdominal mass, intestinal obstruction, gastrointestinal hemorrhage, intra-abdominal sepsis, granulomatous peritonitis and fistulization involving surrounding structures [2]. Clinically, foreign body granuloma may progress, showing various clinical courses depending upon the degree of severity and combinations of these two responses. According to the literature, migration of a retained sponge into the bowel is a rare complication and occurs as a result of inflammation in the intestinal wall that evolves to necrosis [3]. There are only a few case reports of retained surgical sponge penetrating to the intestine in the Japanese literature [4]. We report an unusual case of intestinal obstruction due to complete transmural migration of a retained surgical sponge into the intestine 18 years after gynecological surgery.

\section{Case Report}

A 56-year-old Japanese woman was referred to our hospital in 2009 for colicky abdominal pain, nausea and vomiting which suggested intestinal obstruction. She had undergone gynecological surgery for cervical cancer at another institution 18 years previously. On physical examination her lower abdomen was mildly tender, and bowel sounds were hyperactive. Blood tests showed leukocytosis $\left(10,200 / \mathrm{mm}^{3}\right)$, mild anemia $(10.8 \mathrm{~g} / \mathrm{dl})$ and slightly elevated C-reactive protein $(1.59 \mathrm{mg} / \mathrm{dl})$, while other tests, including tumor markers, were within normal limits.

Contrast-enhanced computed tomography (CT) showed dilation of the small intestine and suggested obstruction in the terminal ileum. At the end of a dilated loop of small intestine, fecal-like material contained tiny air bubbles and small high density areas were observed (ig. 1a). In addition, CT showed a thick-walled cavitary lesion communicating with the proximal jejunum (fig. 1b, fig. 2a). 18F-fluorodeoxyglucose positron emission tomography (FDG-PET) showed abnormal uptake in the left upper abdomen at the same location as the cavitary lesion revealed by CT (fig. 2b). The maximum standardized uptake value of the lesion was 4.2. Although appropriate intravenous fluid supplementation and transnasal long tube decompression were attempted, the ileus did not resolved. The patient underwent laparotomy for the ileus, and the cavitary lesion was resected because malignancy could not be ruled out by FDG-PET findings.

At laparotomy, we found a point of severe obstruction due to adhesion $60 \mathrm{~cm}$ from the terminal ileum. At the end of the dilated loop of small intestine, a hard mass was palpated. After removal of this mass from the intestine, it proved to be a retained surgical sponge (fig. $3 \mathrm{a}$ ). The cavitary tumor which was located in the mesentery of the transverse colon had two fistulae communicating with the proximal jejunum (fig. $3 \mathrm{~b}$ ). The tumor was resected en bloc together with the transverse colon, part of the jejunum and the duodenum. Microscopic examination revealed fibrous encapsulation and foreign body giant cell reaction. The pathological diagnosis was foreign material granulation tissue. After an uneventful postoperative period, the patient was discharged in good general health. 


\section{Discussion}

The reported incidence of retained surgical sponge is one per 1,000-15,000 intra-abdominal operations [1]. While the frequency has probably decreased recently, the true incidence of retained surgical sponge remains uncertain because cases with retained surgical sponges may raise medicolegal issues which promote reluctance to publish actual data. Moreover, some cases remain asymptomatic and may never be discovered. In fact, many cases were discovered to have a retained surgical sponge more than 30 years after the initial surgery [3]. Our present patient had no symptoms during the 18-year period after gynecological surgery.

Zantvoord et al. [3] systematically reviewed 65 published cases of transmural migration of retained surgical sponges, including their own case, based on a PubMed search (1960-2007). They reported that the most common primary operations associated with retained surgical sponges were gynecological (37\%), and that the mean interval between primary surgery and diagnosis of a retained surgical sponge was 93.5 months (10 weeks to 35 years) [2]. The most frequent site of impaction was the intestine (75\%), and most cases (95.3\%) underwent laparotomy to remove the sponge [3]. After complete migration of a retained surgical sponge into the intestine, the sponge usually stops in the terminal ileum, resulting in the type of intestinal obstruction observed in our present case [5]. On the other hand, there are a few case reports describing a retained surgical sponge as being spontaneously expelled rectally [3].

In most of the previously reported cases of complete migration of a retained surgical sponge into the intestine, no fistulae or intestinal wall openings were observed even at the surgical exploration of the abdominal cavity [5, 6], and it is rare for the exact site of migration of a retained surgical sponge into the intestinal lumen to be demonstrated by imaging studies [7]. In our present patient, CT showed a fistula between the cavity and the jejunum. Although it remains unknown whether resection of the cavitary lesion is necessary or not after a retained surgical sponge has been expelled, we resected the lesion in our patient with removal of the retained surgical sponge because a definite preoperative diagnosis could not be obtained in this case. A malignant tumor could not be ruled out based on FDG-PET findings. The only treatment in most of the previously reported cases with complete migration of a retained surgical sponge was removal of the sponge $[5,6]$, though in some case compromised intestine was resected at the same time by laparotomy [5]. However, unlike our patient, none of these cases had evidence of a fistula tract into the intestine, nor of abscesses involving the intestinal wall.

Although FDG-PET findings indicative of a retained surgical sponge are presently limited, a few case reports have described positive findings [8]. It is well known that FDG-PET can show false-positive results for inflammatory granulomas, such as sarcoidosis or tuberculosis. Since the retained surgical sponge in our case produced a foreign body granuloma pathologically, FDG might have accumulated in the granulomatous tissue in our case. Miyake et al. [9] reported FDG-PET findings of 3 cases with foreign body granulomas due to retained textiles and, moreover, reviewed FDG-PET findings of 10 cases from the literature. They described accumulation of FDG as being present in 2 of their 3 cases with foreign body granulomas and in all 10 previously reported cases. Most cases, including their own, showed FDG uptake with a ring-shaped pattern because the retained surgical sponge itself had no FDG 
uptake and FDG activity represented the capsule surrounding the retained surgical sponge [8]. In our case, FDG-PET did not show a ring-shaped pattern because the retained surgical sponge had already been expelled from the capsule.

Although it has been a long time since the routine use of surgical textile materials impregnated with radiopaque markers, a retained surgical sponge can have a long course before clinical manifestation of symptoms. Retained surgical sponges without radiopaque markers remain a possible cause of a disease, as in our present case. Although there are only a few reports of intestinal obstruction due to complete migration of a retained surgical sponge into the intestine $[6,10]$, retained surgical sponge should be considered in the differential diagnosis of intestinal obstruction in patients who have undergone previous abdominal surgery because a retained surgical sponge, especially without radiopaque markers, is difficult to diagnose based on radiological imaging.
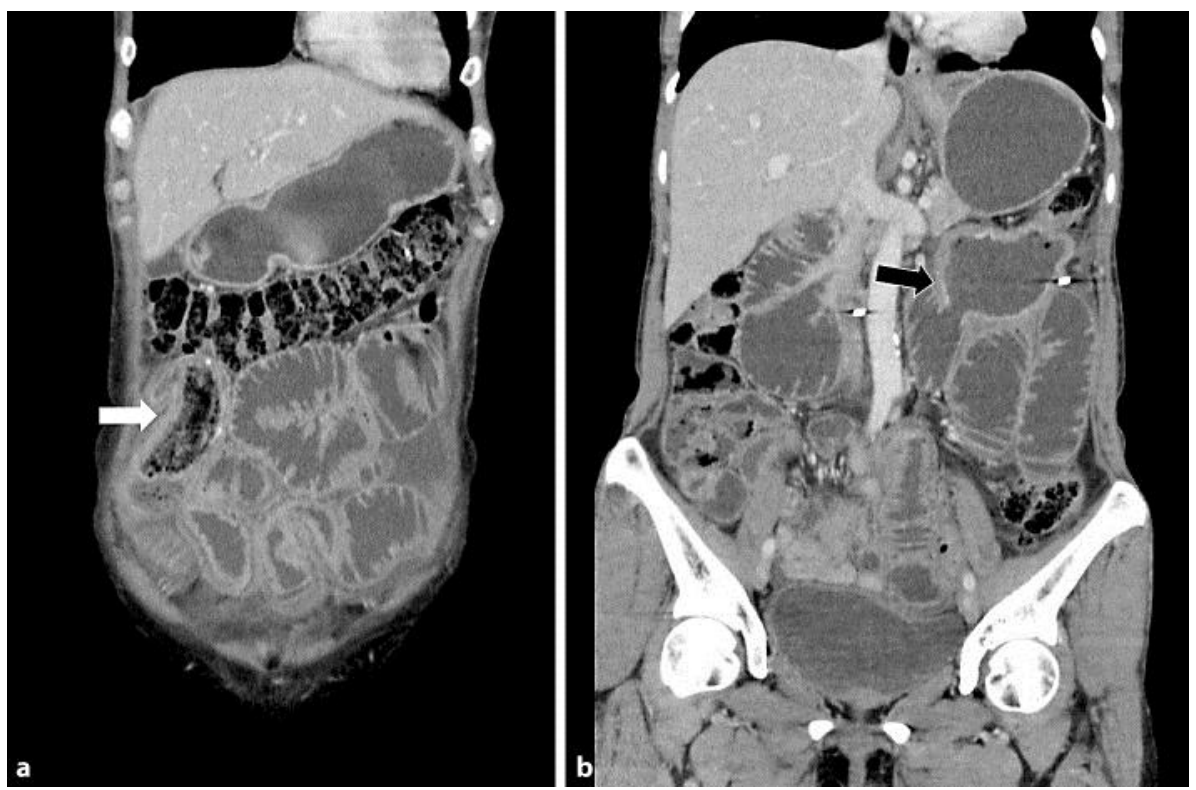

Fig. 1. Coronal CT images. a At the end of a dilated loop of the small intestine, fecal-like masses can be seen (arrow). b CT shows a thick-walled cavitary lesion communicating with the proximal jejunum (arrow). 


\begin{tabular}{r|l|l|l}
$\begin{array}{r}\text { Case Reports in } \\
\text { Gastroenterology }\end{array}$ & $\begin{array}{l}\text { Case Rep Gastroenterol 2012;6:754-759 } \\
\text { DOI: 10.1159/000346285 }\end{array}$ & $\begin{array}{l}\text { Published online: } \\
\text { December 19, 2012 }\end{array}$ & $\begin{array}{l}\text { O 2012 S. Karger AG, Basel } \\
\text { ISSN 1662-0631 } \\
\text { www.karger.com/crg }\end{array}$ \\
\hline
\end{tabular}
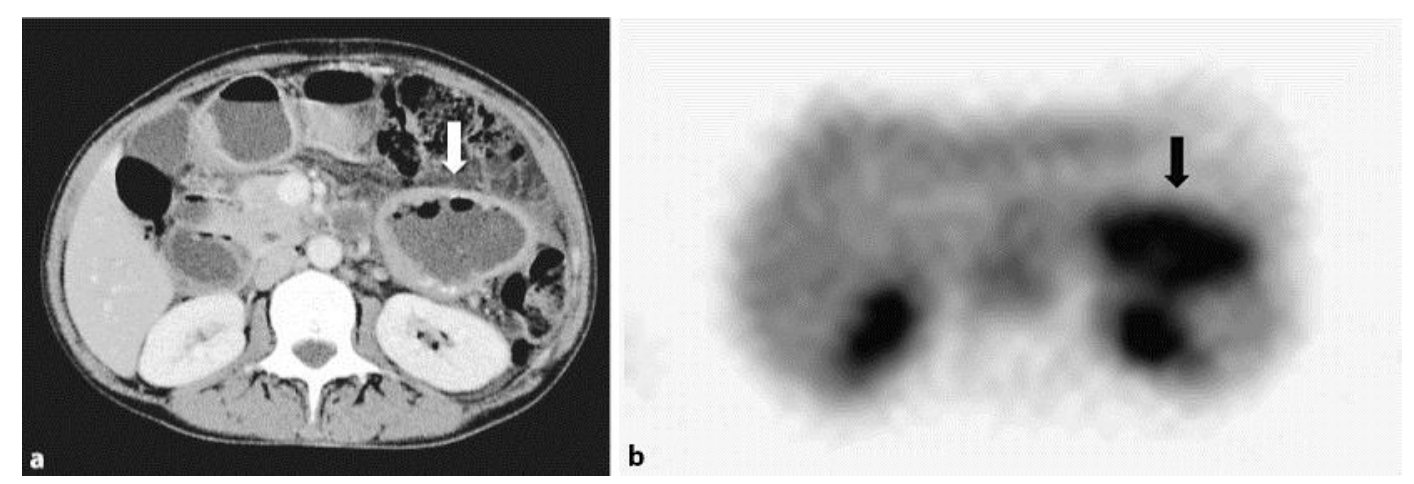

Fig. 2. a Axial CT image shows a thick-walled cavitary lesion $7 \mathrm{~cm}$ in diameter (arrow). b FDG-PET shows abnormal uptake at the same location as the cavitary lesion revealed by CT (arrow).
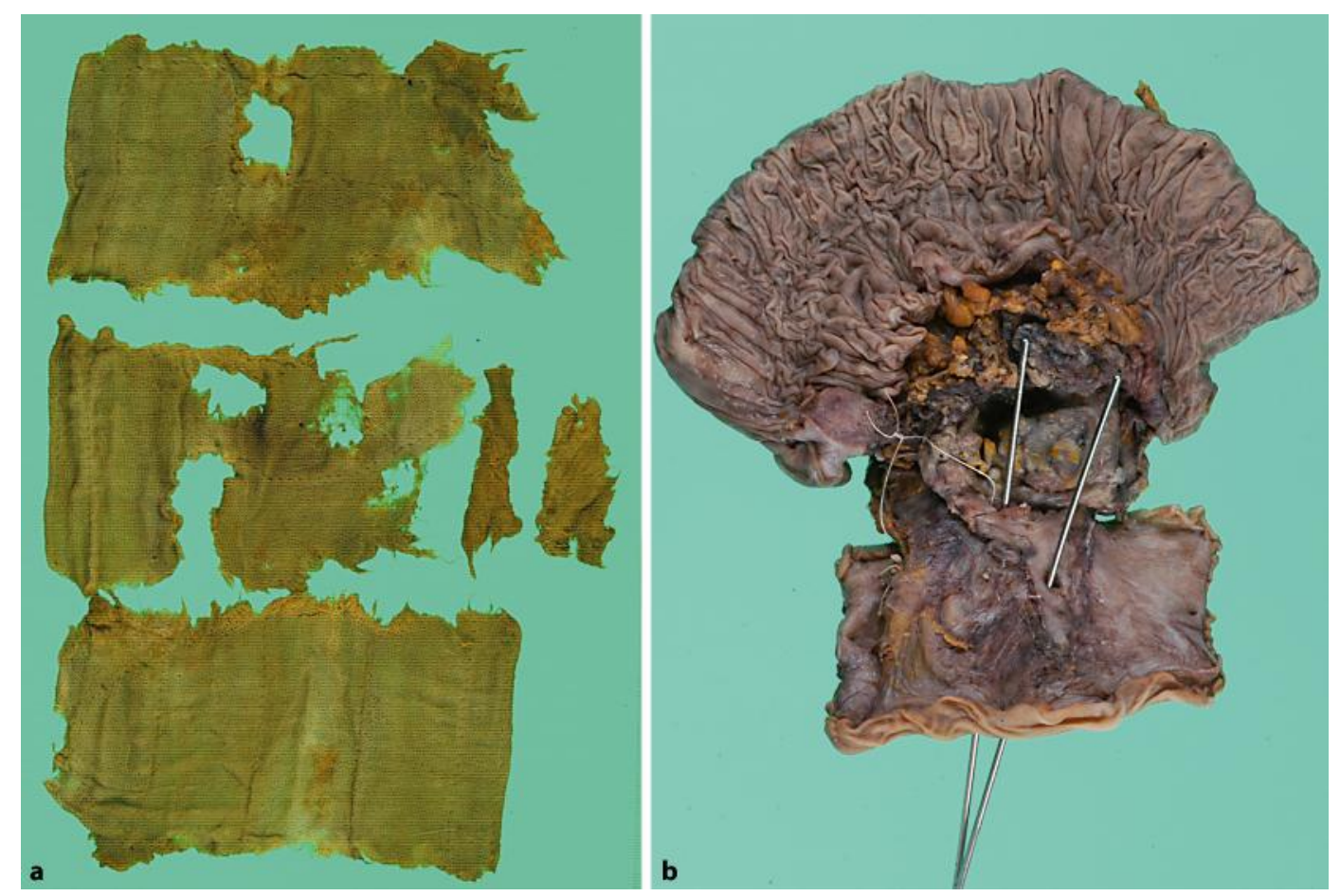

Fig. 3. a The retained surgical sponge removed from the ileum. b Macroscopic findings of the surgical specimen The resected cavitary lesion located in the mesentery of the transverse colon had two fistulae communicating with the proximal jejunum. 


\section{References}

1 Gawande AA, Studdert DM, Orav EJ, Brennan TA, Zinner MJ: Risk factors for retained instruments and sponges after surgery. N Engl J Med 2003;348:229-235.

$\checkmark 2$ Choi BI, Kim SH, Yu ES, Chung HS, Han MC, Kim CW: Retained surgical sponge: diagnosis with CT and sonography. AJR Am J Roentgenol 1988;150:1047-1050.

-3 Zantvoord Y, van der Weiden RM, van Hooff MH: Transmural migration of retained surgical sponges: a systematic review. Obstet Gynecol Surv 2008;63:465-471.

-4 Ko K, Fujioka S, Kato K, Machiki Y, Hashimoto M, Ishikawa A: A case report of a retained gauze sponge in the abdomen with massive bleeding from the rectum. Jpn J Gastroenterol Surg 2000;33:1714-1718.

5 Silva CS, Caetano MR, Silva EA, Falco L, Murta EF: Complete migration of retained surgical sponge into ileum without sign of open intestinal wall. Arch Gynecol Obstet 2001;265:103-104.

-6 Grassi N, Cipolla C, Torcivia A, Bottino A, Fiorentino E, Ficano L, Pantuso G: Trans-visceral migration of retained surgical gauze as a cause of intestinal obstruction: a case report. J Med Case Rep 2008;2:17.

7 Dux M, Ganten M, Lubienski A, Grenacher L: Retained surgical sponge with migration into the duodenum and persistent duodenal fistula. Eur Radiol 2002;12:74-77.

-8 Ghersin E, Keidar Z, Brook OR, Amendola MA, Engel A: A new pitfall on abdominal PET/CT: a retained surgical sponge. J Comput Assist Tomogr 2004;28:839-841.

-9 Miyake KK, Nakamoto Y, Mikami Y, Ishizu K, Saga T, Higashi T, Togashi K: F-18 FDG PET of foreign body granuloma: pathologic correlation with imaging features in 3 cases. Clin Nucl Med 2010;35:853-857.

10 Gencosmanoglu R, Inceoglu R: An unusual cause of small bowel obstruction: gossypiboma - case report. BMC Surg 2003;3:6. 